Sinlo, M. \& Stanier, R. Y. (1957). J. gen. Microbiol. 16, 482-490

\title{
The Utilization of the Tartaric Acids by Pseudomonads
}

\author{
By M. SHILO AND R. Y. STANIER \\ Department of Bacteriology, University of California, Berkeley, California, U.S.A.
}

\begin{abstract}
SUMMARY: Pseudomonas strains, capable of utilizing as carbon sources one or more isomers of tartaric acid, were isolated from soil by specific elective culture methods. Most strains were markedly stereospecific, utilizing only the isomer or isomers upon which they had developed during the elective cultivation. Enzymic study showed that the commonest mechanism for the dissimilation of the tartaric acids by this group of strains is dehydration to oxaloacetic acid, through the action of stereospecific dehydrases. The attack on the tartaric acids is strictly inducible, and in many strains the induced state is rapidly lost in the absence of the substrateinducer. Enzymic assay showed that such loss of induction cannot be accounted for by loss of the specific dehydrase, which is often present in large quantities in organisms which have become incapable of attacking the substrate. This indicates that the metabolism of the tartaric acids requires specific inducible transport systems for bringing the tartaric acids into the cell, in addition to the specific, inducible dehydrases. The existence of such transport systems is further indicated by the fact that the inhibitory interactions between the isomers of tartaric acid, demonstrable with the cell-free dehydrase preparation, do not exist in vivo.
\end{abstract}

Although there has been much work on the utilization of tartaric acids by other groups of micro-organisms, information about the metabolism of these acids by pseudomonads is very limited. Nomura $(1953 a-c)$ and Nomura \& Sakaguchi (1955) reported that a strain of Pseudomonas incognita attacked $d$-tartaric acid* under both aerobic and anaerobic conditions and la Rivière (1956) reported similar findings with another Pseudomonas sp. The isolation of pseudomonads capable of oxidizing the three isomers of tartaric acid has been undertaken and the results are described here.

\section{METHODS}

Unless otherwise specified, the methods employed were those described by Shilo (1957).

Elective cultivation. The elective media used consisted of: $\mathrm{NH}_{4} \mathrm{Cl}, \mathbf{1} \cdot 0 \mathrm{~g}$.; $\mathrm{MgSO}_{4} .7 \mathrm{H}_{2} \mathrm{O}, 0.5 \mathrm{~g}$.; $\mathrm{CaCl}_{2} .2 \mathrm{H}_{2} \mathrm{O}, 0.05 \mathrm{~g}$. $\mathrm{FeCl}_{3}, 0.05$ g.; Yeast extract (Difco), 0.05 g.; phosphate buffer $(\mathrm{M} / \mathbf{1 5 0}, \mathrm{pH} \mathrm{7 \cdot 2),} 1 \mathrm{l}$. This basal medium was supplemented with $1 \mathrm{~g}$. of an isomer of tartaric acid. Shallow layers of liquid medium in Erlenmeyer flasks were inoculated with soil and incubated at $30^{\circ}$ without agitation. After 24-48 hr., transfers were made to second flasks containing media of the same composition. After growth had occurred in the second flasks, streaks were made on plates of the same medium solidified with agar. Experience showed that the organisms isolated by enrichment with one isomer of tartaric acid were in general not able to attack the other isomers.

* For a discussion of the configurational notation employed, see Shilo (1957). 
In order to obtain strains capable of attacking two or three isomers of tartaric acid, the elective procedure was slightly modified. After an elective culture had been established with one isomer, a transfer was made to a flask of similar medium with a second isomer, and this, in turn, was transferred after growth to a flask of elective medium with the third isomer. By such successive elective cultures, it was easy to obtain strains which attacked two or three isomers of tartaric acid.

\section{RESULTS}

Table 1 shows the patterns of utilization of the tartaric acids and related acids as carbon and energy sources for growth by the pseudomonads which were isolated. Where the pathway of utilization of tartaric acid has been established through enzymic study, the nature of this pathway is indicated. It can be seen that attack on the tartaric acids by dehydration to oxaloacetic acid is the most common pathway in this group, although there are two strains for which a different pathway is indicated from preliminary enzymic observations. A very striking feature of the patterns of utilization shown in Table 1 is the specificity of many strains with respect to utilization of the three tartaric acids. Two sets of elective cultures with either $d$ - or meso-tartrate yielded only strains capable of attacking the isomer used for election; in one elective culture with the $l$-isomer, strains capable of growth on both enantiomorphs were found.

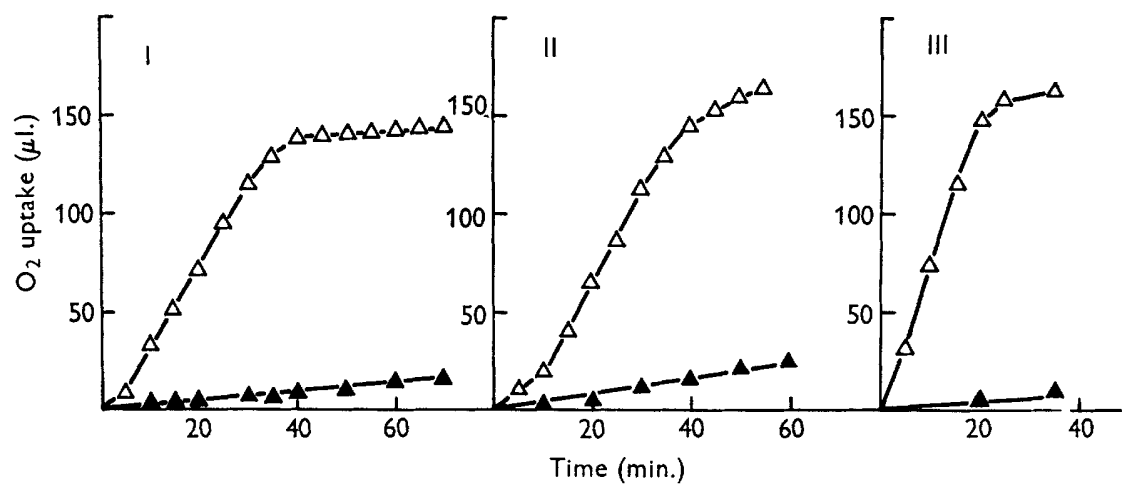

Fig. 1. The specificity of oxidation of the tartaric acids by three pseudomonad strains. Oxygen uptakes by suspensions furnished with $5 \mu$ mole substrate. (I) Strain $l 1$, grown on $l$-tartrate. $\Delta-\triangle, l$-tartrate; $\Delta-\Delta$, endogenous, $d$-tartrate, meso-tartrate. (II) Strain $d$ 15, grown on $d$-tartrate. $\triangle-\triangle$, $d$-tartrate; $\Delta--\Delta$, endogenous, $l$-tartrate, meso-tartrate. (III) Strain meso 1 , grown on meso-tartrate. $\triangle-\triangle$, mesotartrate; $\boldsymbol{\Delta}-\boldsymbol{\Delta}$, endogenous, $l$-tartrate, $d$-tartrate.

\section{Pattern of induction}

In every case, the attack on the tartaric acids was a strictly inducible one: the tartrate-utilizing strains were incapable of attacking tartaric acid immediately after growth on succinate or acetate. Fig. 1 shows the specificity of attack on the tartaric acids by strains which were isolated through elective culture with meso-, $l$ - and $d$-tartrate, respectively. In this experiment, each strain had been grown at the expense of the isomer of tartaric acid used for its election. 


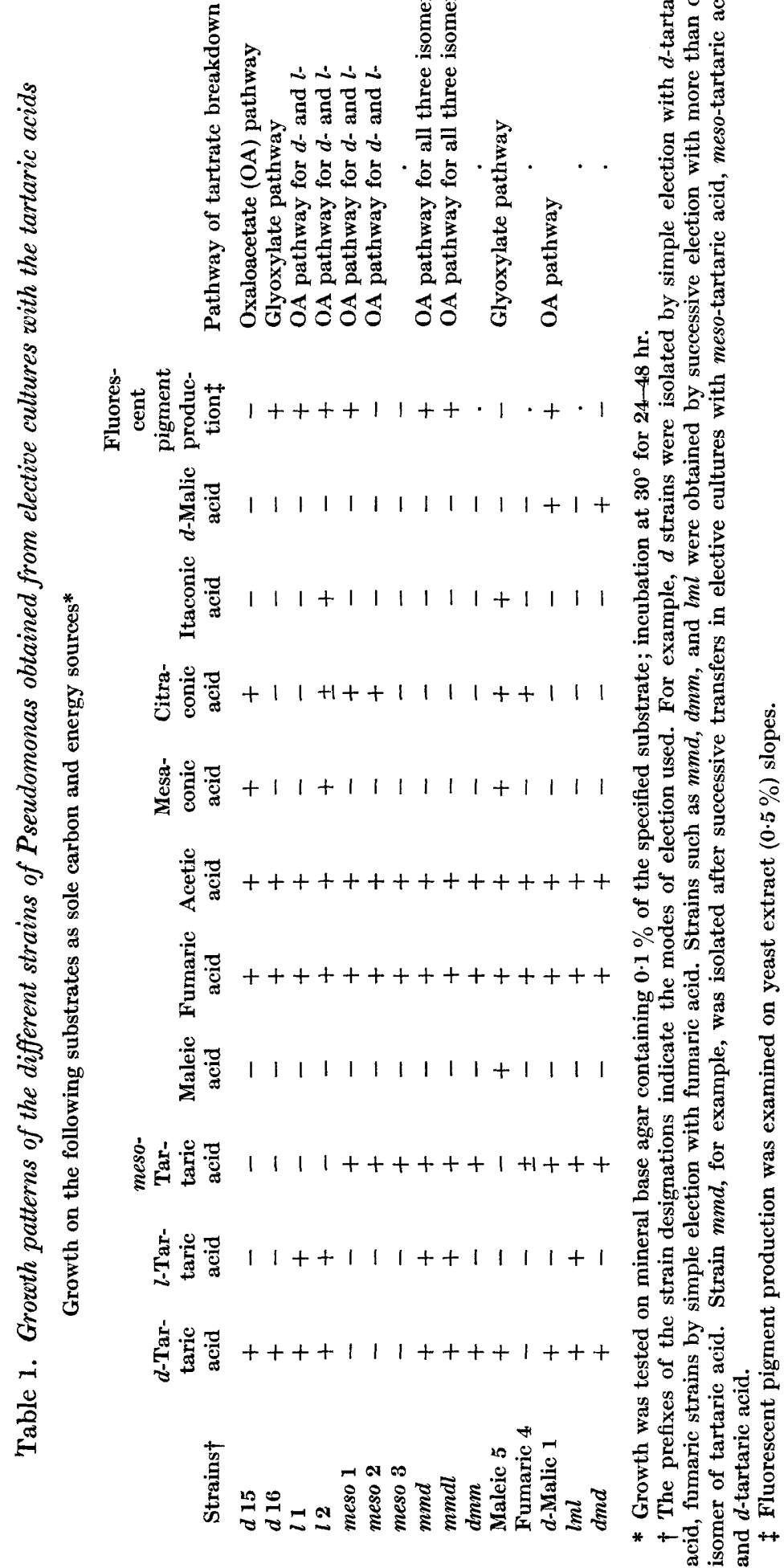


It will be noted that strain $l 1$, although capable of growth at the expense of either enantiomorph, did not immediately attack the $d$-isomer after growth on $l$-tartaric acid. When this strain was tested for the utilization of the enantiomorphs after growth on $d$-tartaric acid, there was an immediate rapid attack on the $d$-isomer and a very weak, but significant, attack on the $l$-isomer. In all cases, the total oxygen uptake/mole tartrate oxidized was slightly more than 1 mole, i.e. about $40 \%$ of the theoretical value for complete oxidation. This probably reflected the occurrence of oxidative assimilation.

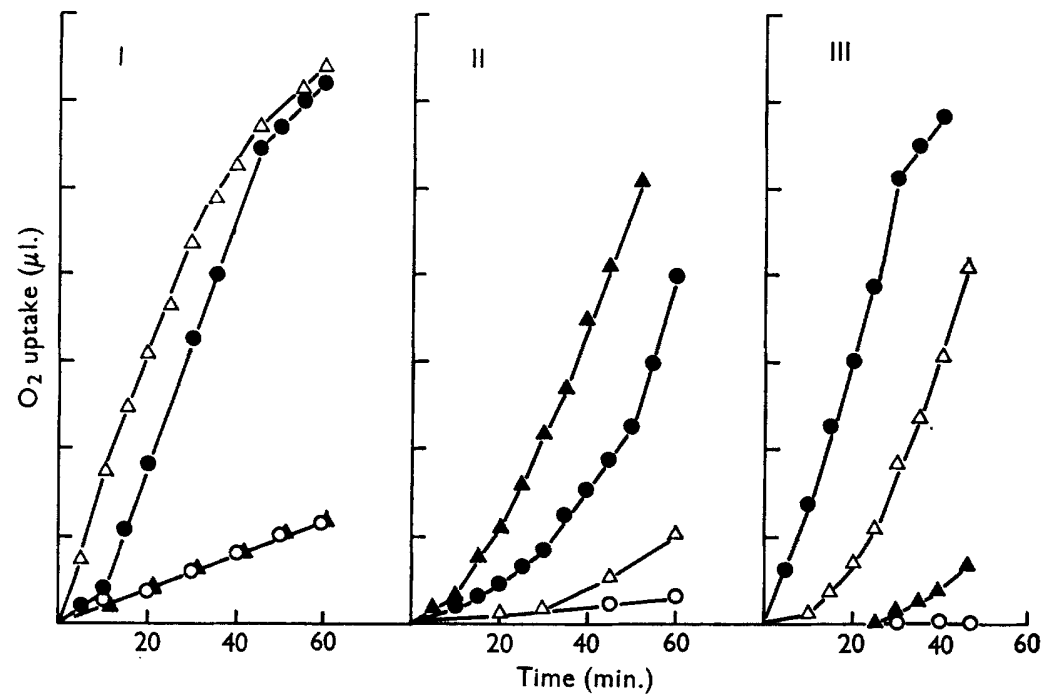

Fig. 2. Patterns of induction for oxidation of the tartaric acids by a pseudomonad strain $(\mathrm{mmdl}$ ) capable of utilizing all three isomers. (I), grown on $l$-tartrate; (II), grown on $d$-tartrate; (III), grown on meso-tartrate. $\mathrm{O}-\mathrm{O}$, endogenous; $\Delta-\Delta, 10 \mu$ mole $d$-tartrate; $\triangle-\triangle, 10 \mu$ mole $l$-tartrate; - , $10 \mu$ mole meso-tartrate.

In Fig. 2, the pattern of oxidation of the tartaric acids by a strain capable of growing on all three isomers is shown. The specificity of induction by the isomer used for growth is evident. There is one curious feature of the induction patterns : growth on meso-tartaric acid appears greatly to accelerate induction for the $l$-isomer, and vice versa. This effect cannot be explained by non-specific induction; extracts of cells grown on the meso-isomer do not contain $l$-tartaric acid dehydrase, and extracts of the cells grown on the $l$-isomer do not contain meso-tartaric acid dehydrase.

\section{The loss of induction}

Early in the course of experiments on inductive patterns, it was observed that induction for the attack on $d$ - and meso-tartrate was very readily lost. This phenomenon was most easily observed in cultures grown with limiting amounts of tartrate. Since tartrate was the sole source of carbon and energy, its depletion caused the population to enter the stationary phase, and this was invariably followed by a loss of the capacity for immediate attack on tartrate. The later the organisms are harvested in the stationary phase, the greater 
becomes the lag before tartrate can be oxidized at a rapid rate. For example, organisms which have remained in the stationary phase for $4 \mathrm{hr}$. after tartrate depletion show, when harvested and tested manometrically, an absolute lag of $20 \mathrm{~min}$. before tartrate is oxidized at all, and oxidation at maximal rate is established only after 50-60 min. (Fig. 3). It can easily be shown, by testing the dehydrase activity of extracts from these organisms, that the inability to attack tartrate is not a reflexion of the complete loss of the specific dehydrase. Organisms which have remained in the stationary phase for $4 \mathrm{hr}$. still contain $50 \%$ of the specific dehydrase activity found in the population at the end of the logarithmic phase of growth (Table 2). On the basis of the enzymic analysis, we would expect a diminution in the rate of tartrate oxidation by whole organisms, but not a total loss. Since, however, the primary step in the

Table 2. The behaviour of whole organisms of Pseudomonas strain $m 1$ with respect to the oxidation of meso-tartrate, and the meso-tartrate dehydrase activity shown by extracts prepared from them.

Data on whole organisms taken from Fig. 3. Dehydrase assays were performed as described by Shilo (1957), and specific activities of extracts were computed on the basis of protein content.

\begin{tabular}{|c|c|c|c|c|c|}
\hline & & Whole cell & & & \\
\hline & & & $\begin{array}{l}\text { Effect of an } \\
\text { equimolar }\end{array}$ & of mes & $\begin{array}{l}\text { draric acid } \\
\text { drase }\end{array}$ \\
\hline $\begin{array}{c}\text { Age of culture } \\
\text { at time of } \\
\text { harvest } \\
\text { (hr.) }\end{array}$ & $\begin{array}{l}\text { Initial rate } \\
\text { of meso- } \\
\text { tartrate } \\
\text { oxidation, } \\
\mu \mathrm{l} . \mathrm{O}_{2} / \mathrm{hr} .\end{array}$ & $\begin{array}{l}\text { Duration of } \\
\text { lag before } \\
\text { initiation of } \\
\text { oxidation } \\
\text { (min.) }\end{array}$ & $\begin{array}{l}\text { quantity of } \\
d \text {-isomer on } \\
\text { rate of meso- } \\
\text { tartrate } \\
\text { oxidation }\end{array}$ & $\begin{array}{c}\text { No } \\
d \text {-isomer } \\
\text { present }\end{array}$ & $\begin{array}{c}\text { Equimolar } \\
\text { quantity of } \\
d \text {-isomer } \\
\text { present }\end{array}$ \\
\hline $10 \cdot 5$ & 200 & 0 & None & 100 & 20 \\
\hline $14 \cdot 5$ & 0 & 20 & None & 48 & 11 \\
\hline $18 \cdot 5$ & 0 & 40 & None & 25 & 7 \\
\hline
\end{tabular}

attack on tartrate is a non-oxidative one, it might be imagined that the total loss of the ability to consume oxygen at the expense of tartrate reflects the loss of the ability to attack the first oxidizable intermediate, even though tartrate itself can still be broken down. This explanation could be excluded by the demonstration that such organisms can immediately consume oxygen when furnished with oxaloacetate or pyruvate, the demonstrated metabolic intermediates in the dissimilation of tartrate. Clearly, therefore, the observed loss of induction cannot be explained on the basis of the loss of any of the known enzyme systems involved in the dissimilatory pathway for tartrate. It seems necessary to postulate the existence of an additional inducible system, required for transporting exogenous tartrate to the intracellular site of dehydrase action.

\section{Discrepancies between inhibitory patterns shown in extracts and whole organisms}

Further evidence for the assumption that the tartaric acids cannot freely penetrate from the environment to the site of dehydration without a preceding specific induction comes from the study of specific inhibition. As shown in the 
accompanying paper by Shilo (1957), the meso-isomer of tartaric acid is a powerful inhibitor of the $d$ dehydrase, and the $d$-isomer of the meso dehydrase. Yet when these inhibitors are tested with whole organisms, no inhibition, as measured in terms of oxygen uptake, can be shown. The absence of inhibition of the oxidation of meso-tartrate by the $d$-isomer in vivo is shown in Fig. 3, while Table 2 shows the marked inhibition (about $80 \%$ ) of the meso dehydrase by the $d$-isomer which occurs in extracts from these same organisms.

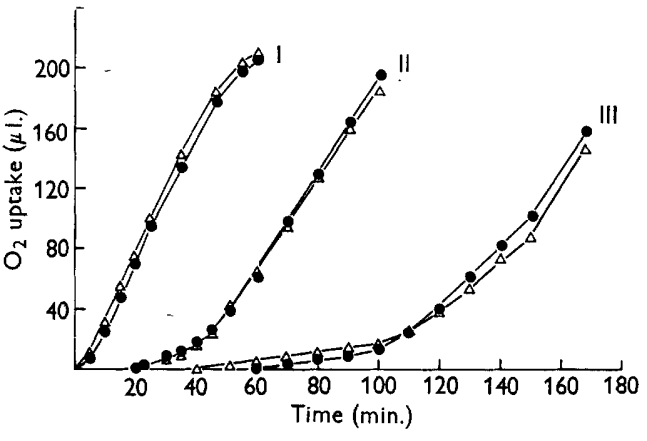

Fig. 3

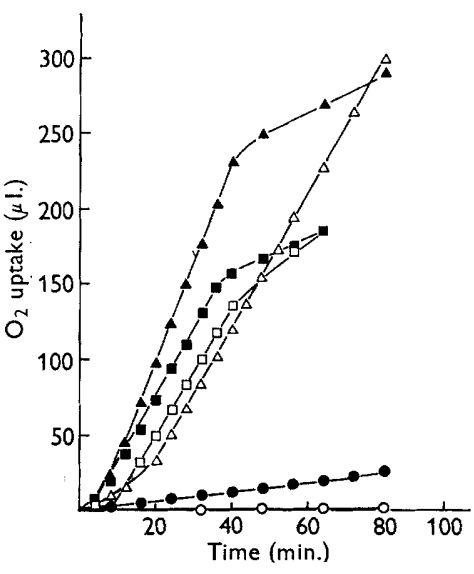

Fig. 4

Fig. 3. The oxidation of meso-tartrate by washed pseudomonads of strain $m 1$, harvested at various times from a medium containing meso-tartrate as sole carbon source. The culture entered the stationary phase at about $10.5 \mathrm{hr}$., because of meso-tartrate depletion. (I), organisms harvested at $10.5 \mathrm{hr}$; (II) harvested at $14.5 \mathrm{hr}$; (III) harvested at $18.5 \mathrm{hr}$. - oxygen uptake with meso-tartrate $(10 \mu$ mole $) ; \triangle-\triangle$, oxygen uptake with meso-tartrate $(10 \mu$ mole $)+d$-tartrate $(10 \mu$ mole $)$. Endogenous respiration was negligible. Note the pronounced absolute lags in substrate oxidation after $14.5 \mathrm{hr}$. and $18.5 \mathrm{hr}$., and the absence of inhibition of meso-tartrate oxidation by $d$-tartrate.

Fig. 4. The inhibitory effect of $d$-tartrate on the oxidation of meso-tartrate by washed organisms of strain mmdl grown at the expense of meso-tartrate. $\mathrm{O}-\mathrm{O}$, endogenous respiration; $\mathbf{\square - \square}$, meso-tartrate $(5 \mu \mathrm{mole}) ; \square-\square], \quad l$-tartrate $(5 \mu \mathrm{mole}) ; \boldsymbol{\Delta}-\boldsymbol{\Delta}$, meso-tartrate $(5 \mu$ mole $)+l$-tartrate $(5 \mu$ mole $)$; - meso-tartrate $(5 \mu$ mole $)+d$-tartrate $(5 \mu$ mole $) ; \triangle-\triangle, d$-tartrate $(5 \mu$ mole $)+l$-tartrate $(5 \mu$ mole $)$.

\section{Inhibition of tartrate utilization by ultraviolet irradiation}

The experiments reported above indicate that the tartaric acids cannot freely reach the intracellular sites of their breakdown by simple diffusion from the medium in which the organisms are suspended. However, the analysis of inductive patterns suggests that exposure to tartaric acid somehow changes the cells so that these substances can freely enter them. It is therefore tempting to assume that a special inductive event, the development of a specific carrier system, takes place when the cells are exposed to tartrate. In support of this assumption it has been observed that when organisms, harvested from a tartrate-grown culture in the stationary phase, are exposed to brief ultraviolet irradiation, they become almost completely incapable of regaining the ability 
to oxidize tartrate. The dose of radiation employed had no effect on preformed enzyme systems, as shown by its inability to affect the oxidation of pyruvate. Since it is well known that ultraviolet irradiation has a highly selective inhibitory effect on enzyme synthesis, these findings suggest that a de novo act of enzyme formation is required in order for the cells to regain the ability to attack tartrate.

\section{Inhibitory interactions in whole cells}

In one strain ( $\mathrm{mmdl}$ ), it was possible to demonstrate the inhibition of the oxidation of meso-tartrate in vivo. The strain concerned can attack all three isomers of tartaric acid. When the organisms have been grown at the expense of meso-tartrate, the oxidation of this isomer can be almost totally inhibited by the simultaneous addition of an equimolar amount of the $d$-isomer (Fig. 4). However, the expected inhibition by the meso-isomer of the attack on the enantiomorphs, which is readily demonstrable with extracts of this strain, is not observed with whole organisms; the oxidations of $d$ - and of $l$-tartaric acids by specifically induced organisms are unaffected by the presence of the mesoisomer. These observations can be explained by assuming that strain $m m d l$ has a constitutive system for the transport of the $d$-isomer into the cell, but not for the transport of the meso-isomer. The dehydrases are all clearly inducible.

\section{DISCUSSION}

In the accompanying paper (Shilo, 1957) it was shown that the dissimilation of the tartaric acids by various pseudomonads involves the action of dehydrases, stereospecific with respect to substrate and inducer : both substrate and inducerfunction for each dehydrase are shown by one, and only one, isomer of the tartaric acids. The experiments with whole organisms reported in this paper reveal, however, that the acquisition of the ability to attack any one of the isomers of tartaric acid is not solely a reflexion of the induced synthesis of the relevant dehydrase; dehydrase synthesis is a necessary, but not sufficient, event. It is evident that each isomer of tartaric acid also induces specifically the formation of a system which is required for the transport of the isomer in question from the external environment to the site of dehydrase action within the cell.

The specific induction of transport systems, required to bring substances to to the site of enzyme action in bacteria, has been clearly established by a number of earlier investigators. Barrett \& Kallio (1953) showed that the "adaptive lag' in the utilization of exogenous citrate by Pseudomonas fuorescens cannot be explained by the lack of the relevant enzymes of the tricarboxylic acid cycle, which are present in extracts from cells 'unadapted' to oxidize citrate. Nor is the lag a reflexion of the existence of a mechanical permeability barrier, since Barrett, Larsen \& Kallio (1953) found that inhibitors of enzyme synthesis (ultraviolet irradiation, amino acid analogues) could totally block the induction of ability to oxidize citrate. The fact that inhibition by amino acid analogues can be prevented by simultaneous addition to the system 
of the relevant amino acid speaks particularly strongly for the necessity of a specific act of enzyme synthesis to make externally supplied citrate available to the intact organism. A more detailed study of the process of citrate utilization in Aerobacter aerogenes (Davis, 1956) revealed a similar situation. In $A$. aerogenes, it has been directly demonstrated that the intracellular accumulation of citrate occurs only after specific induction. Indeed, it seems very probable that much of the evidence obtained with whole organisms, which has tended to suggest that the tricarboxylic acid cycle is not functional in aerobic micro-organisms, can be best interpreted as reflecting the existence of barriers, only overcome by specific induction, which bar the entry into the cell of exogenous tricarboxylic acid cycle components.

Specific transport mechanisms in micro-organisms for other exogenous nutrilites have been shown. Thus, in Escherichia coli an inducible transport system clearly controls the entry of $\beta$-galactosides into the cell, and this system has provided the material for the most detailed and elegant experiments so far done on the general phenomenon (Monod, 1956; Dr Melvin Cohn, personal communication). Specific constitutive transport systems for the accumulation of various amino acids in bacteria have been shown (Cohen $\&$ Rickenberg, 1955). Indeed, the now rapidly accumulating evidence suggest that most of the nutrilites which the bacteria absorb from their environment must gain entry to the cell by a process of active transport, rather than of simple diffusion. The inducible control exerted over so many of these transport mechanisms is clearly of very great adaptive significance, since it confers on the microbial cell a high degree of plasticity and selectivity in the assimilation of compounds from its ever-changing external environment.

The work described in this paper was performed while the senior author held a Judah L. Magnes Fellowship, during leave from the Laboratory for Microbiological Chemistry, Hebrew University, Hadassah Medical School, Jerusalem, Israel. It was aided by a grant from the cancer research funds of the University of California.

\section{REFERENCES}

Barrett, J. T. \& Kallio, R. E. (1953). Terminal respiration in Pseudomonas fluorescens: component enzymes of the tricarboxylic acid cycle. J. Bact. 66, 517 .

BarretT, J. T., Larson, A. D. \& Kallio, R. E. (1953). The nature of the adaptive lag of Pseudomonas fluorescens toward citrate. J. Bact. 65, 187.

Cohen, G. N. \& RickenberG, H. V. (1955). Existence d'accepteurs spécifiques pour les aminoacides chez Escherichia coli. C.R. Acad. Sci., Paris, 240, 2086.

Davis, B. D. (1956). Relations between enzymes and permeability (membrane transport) in bacteria. In Enzymes, Units of Biological Structure and Function, p. 509. New York: Academic Press.

MoNoD, J. (1956). Remarks on the mechanism of enzyme induction. In Enzymes, Units of Biological Structure and Function, p. 7. New York: Academic Press.

Nomura, M. $(1953 a)$. The decomposition of d-tartarate by the micro-organism. J. agric. chem. Soc. Japan, $27,46$.

Nomura, M. $(1953 b)$. The decomposition of d-tartarate by the micro-organism. II. On the anaerobic decomposition of d-tartarate in the presence of radioactive bicarbonate. J. agric. chem. Soc. Japan, 27, 554. 
Nomura, M. $(1953 c)$. The decomposition of $\mathrm{L}-(+)$-tartarate by the micro-organism. III. On the anaerobic metabolism of phosphoglycerate and oxalacetate as a possible intermediate in the anaerobic decomposition of tartarate by Pseudomonas incognita. J. agric. chem. Soc. Japan, 27, 860.

Nomura, M. \& Sakaguchi, K. (1955). 'The decomposition of L ( + ) tartrate by microorganisms. J. gen. appl. Microbiol. (Japan), 1, 77.

Rivière, J. W. M. LA (1956). Intermediate products in tartrate decomposition by cell-free extracts of Pseudomonas putida under anaerobic conditions. Biochim. biophys. Acta, 21, 190.

Shrlo, M. (1957). The enzymic conversion of the tartaric acids to oxaloacetic acid. J. gen. Microbiol. 16, 472.

(Received 24 October 1956) 